\title{
Dynamical Negative Differential Resistance in Antiferromagnetically Coupled Few-Atom Spin Chains
}

\author{
Steffen Rolf-Pissarczyk, ${ }^{1,2,{ }^{*}}$ Shichao Yan, ${ }^{1,2,3, \dagger}$ Luigi Malavolti, ${ }^{1,2}$ \\ Jacob A. J. Burgess, ${ }^{1,2,4}$ Gregory McMurtrie, ${ }^{1,2}$ and Sebastian Loth ${ }^{1,2,5, \$}$ \\ ${ }^{1}$ Max Planck Institute for the Structure and Dynamics of Matter, Luruper Chaussee 149, 22761 Hamburg, Germany \\ ${ }^{2}$ Max Planck Institute for Solid State Research, Heisenbergstraße 1, 70569 Stuttgart, Germany \\ ${ }^{3}$ School of Physical Science and Technology, ShanghaiTech University, Shanghai 201210, China \\ ${ }^{4}$ Department of Physics and Astronomy, University of Manitoba, Winnipeg, Manitoba R3T 2N2, Canada \\ ${ }^{5}$ University of Stuttgart-Institute for Functional Matter and Quantum Technologies, Pfaffenwaldring 57, 70569 Stuttgart, Germany
}

(Received 18 March 2017; revised manuscript received 2 October 2017; published 21 November 2017)

\begin{abstract}
We present the appearance of negative differential resistance (NDR) in spin-dependent electron transport through a few-atom spin chain. A chain of three antiferromagnetically coupled Fe atoms (Fe trimer) was positioned on a $\mathrm{Cu}_{2} \mathrm{~N} / \mathrm{Cu}(100)$ surface and contacted with the spin-polarized tip of a scanning tunneling microscope, thus coupling the Fe trimer to one nonmagnetic and one magnetic lead. Pronounced NDR appears at the low bias of $7 \mathrm{mV}$, where inelastic electron tunneling dynamically locks the atomic spin in a long-lived excited state. This causes a rapid increase of the magnetoresistance between the spin-polarized tip and Fe trimer and quenches elastic tunneling. By varying the coupling strength between the tip and Fe trimer, we find that in this transport regime the dynamic locking of the Fe trimer competes with magnetic exchange interaction, which statically forces the Fe trimer into its high-magnetoresistance state and removes the NDR.
\end{abstract}

DOI: 10.1103/PhysRevLett.119.217201

Spintronics utilizes the electron spin as a further degree of freedom in solid-state devices. New spintronic applications in magnetic sensing, data storage, and quantum information processing are enabled [1-3] because of the low power needed to manipulate spins compared to chargebased electronics. Due to this, there is great interest in translating general functionalities of conventional chargebased electronics into a spin-based footing.

Negative differential resistance (NDR), referring to a decrease in current with increasing voltage, is an important effect that enables a two-terminal device to operate as an amplifier or oscillator. It is usually achieved by the shift or occupation of electronic states in semiconducting tunnel junctions [4,5], transport through atoms, molecules, and quantum dots [6-11], or recently the charging dynamics of dopants [12].

Spin-based NDR has been reported for electron transport through single or coupled quantum dot devices [13-15] as well as through single-molecule magnets involving nondegenerate spin multiplets [16]. It has been proposed that spin-based NDR, purely based on spin-spin interaction, will appear in inelastic electron transport through anisotropic magnetic systems coupled to one ferromagnetic lead and one nonmagnetic lead [17], but this effect has hitherto not been observed experimentally.

Here we report the appearance of spin-based NDR in inelastic electron transport through an anisotropic antiferromagnetically coupled few-atom spin chain in a spin-polarized scanning tunneling microscope (STM). In this configuration, the spin-polarized tip acts as the magnetic lead with a precisely adjustable position and the sample substrate as the nonmagnetic lead on which the spin chain directly lies. Inelastic scattering of electrons locks the spin chain into an excited spin state with a large magnetoresistance. This suppresses elastic tunneling between the ferromagnetic lead and spin chain, causing NDR at a very low voltage. By varying the STM tip-sample distance, we adjust the coupling strength between the spin-polarized tip and spin chain and measure variations in the NDR. We find that electron tunneling competes with magnetic exchange interaction between the spin-polarized tip and spin chain that occurs when bringing the spin chain into contact with the spin-polarized tip. For weak coupling, the dynamic locking of the spin chain produces prominent NDR, whereas for strong coupling, the magnetic exchange interaction forces the spin chain into a spin configuration that no longer permits NDR.

The experimental setup is depicted in Fig. 1(a). The fewatom spin chain is sandwiched between the tip of an STM and a $\mathrm{Cu}$ substrate [Fig. 1(a)]. The $\mathrm{Cu}$ surface was passivated by a monolayer of copper nitride $\left(\mathrm{Cu}_{2} \mathrm{~N}\right)$ prior to the experiment, and the spin chain was assembled by placing three $\mathrm{Fe}$ atoms (Fe trimer) at a spacing of $0.72 \mathrm{~nm}$ on $\mathrm{Cu}$ binding sites of the $\mathrm{Cu}_{2} \mathrm{~N}$ layer [18-21]. A spin polarization of the STM tip of $\eta_{t}=0.6$ was achieved by picking up several $\mathrm{Fe}$ atoms which remain at the tip apex and applying an external magnetic field of up to $2 \mathrm{~T}$ [22-24]. This geometry effectively creates a doublebarrier structure into which the $\mathrm{Fe}$ trimer is embedded with one spin-polarized and one nonpolarized electrode 


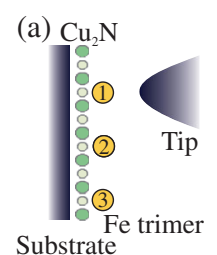

(e)

(g)

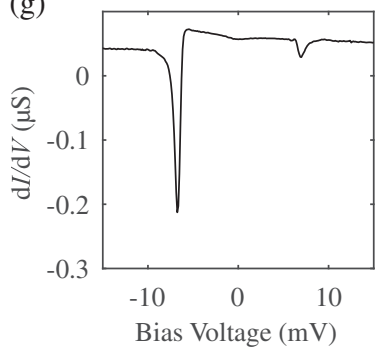

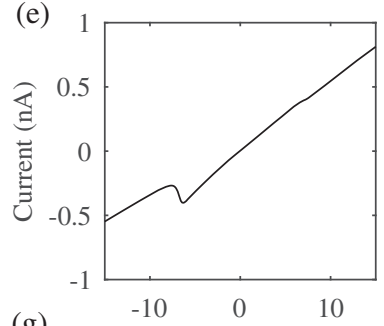

(b) $|+|$ (c)

(c)

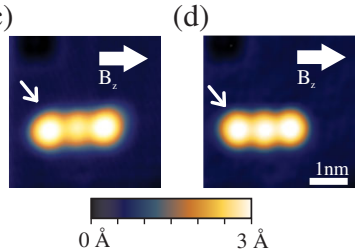

(f)

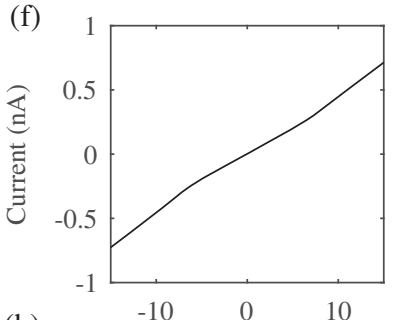

(h)

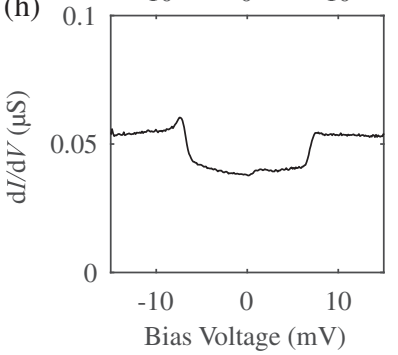

FIG. 1. (a) Schematic of the experimental setup. (b) $\mathrm{The}_{2} \mathrm{Cu}_{2} \mathrm{~N}$ layer and the vacuum form a two-barrier structure around the $\mathrm{Fe}$ trimer. (c), (d) STM constant-current topographies of a few-atom spin chain measured with a spin-polarized tip (c) and a non-spinpolarized tip (d) at a tunnel junction set point of $2 \mathrm{nS}$ with $5 \mathrm{mV}$. (e), (f) $I(V)$ curves recorded with a spin-polarized tip (e) and with a non-spin-polarized tip (f) on the side atom of the Fe trimer [indicated as small white arrows in (c) and (d)]. The tunnel junction set point is $54 \mathrm{nS}$ at $+15 \mathrm{mV}$, and the external field is $1 \mathrm{~T}$. $(\mathrm{g})$, (h) $d I / d V$ spectra recorded with a spin-polarized tip (g) and a nonspin-polarized tip (h) recorded simultaneously with the $I(V)$ curves shown in (e) and (f).

[Fig. 1(b)]. At a low temperature $(0.5 \mathrm{~K})$, this enables long lifetimes of spin excitations in the Fe trimer, ranging up to microseconds [20], such that tunnel currents as low as a picoampere will lead to nonequilibrium transport conditions in this spin system.

With a spin-polarized tip, the $I(V)$ curve recorded above a side atom of the Fe trimer shows Ohmic behavior at a small bias and a pronounced nonlinearity at $-6.7 \mathrm{mV}$ [Fig. 1(e)]. Between -6 and $-7.5 \mathrm{mV}$, the current drops with increasing bias amplitude. This clear signature of NDR leads to a pronounced dip in the differential conductance $(d I / d V)$ spectrum [Fig. $1(\mathrm{~g})$ ]. At a positive bias, a similar, but weaker, dip is observed at $+6.7 \mathrm{mV}$.

By contrast, the $I(V)$ curve recorded with a non-spinpolarized tip is more symmetric on the positive and negative bias sides and shows no NDR [Fig. 1(f)]. Instead, it features kinks at +6.8 and $-6.8 \mathrm{mV}$, which result in steps in the $d I / d V$ spectrum [Fig. 1(h)]. The absence of NDR for a non-spin-polarized current demonstrates that the NDR is not caused by moving a localized electronic state through the transport channel as found for tunneling through molecules [26], nonmagnetic clusters, or dopants [12]. It must instead be caused by a spin-dependent effect which links to the magnetic states of the Fe trimer.

The steps in the $d I / d V$ spectrum recorded with a nonspin-polarized tip indicate the onset of significant inelastic tunneling at $6.8 \mathrm{meV}$ that can promote the Fe trimer into excited spin states $[27,28]$. This is coincident with the voltage position of the NDR in the spin-polarized $d I / d V$ spectrum. To determine whether the observed NDR is indeed caused by dynamic processes $[18,29]$ induced by inelastic electron transport, we performed conductancedependent measurements with the spin-polarized tip. $I(V)$ curves were recorded with successively increasing set point conductance [Fig. 2(a)]. We characterize the strength of the NDR feature by the peak-to-valley current ratio (PVR) [5], as shown in Fig. 2(a). Starting at a junction conductance of $0.01 \mu \mathrm{S}$ (referenced at $+15 \mathrm{mV}$ ), we find that the magnitude of the NDR grows as the conductance increases up to a value of $0.27 \mu \mathrm{S}$, achieving a maximum value $\mathrm{PVR}=$ 1.61. For even larger conductances, the PVR breaks down at $0.43 \mu \mathrm{S}$ and diminishes until the NDR feature vanishes at a conductance of $0.95 \mu \mathrm{S}$ [Fig. 2(b)].

These conductance-dependent measurements clearly link the NDR to a dynamic effect induced by the tunneling electrons with an energy close to or at the inelastic tunneling threshold. It is therefore likely that it relates to the dynamic interplay between the spin-state occupation of

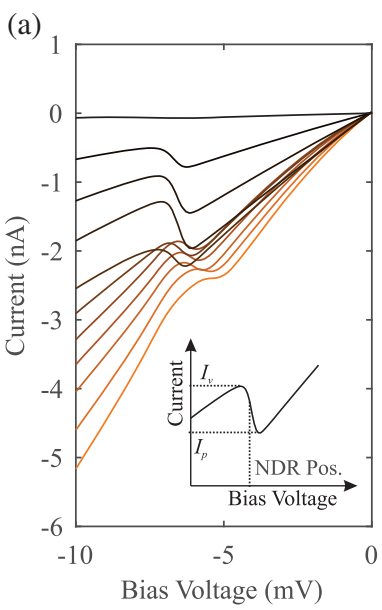

(b)
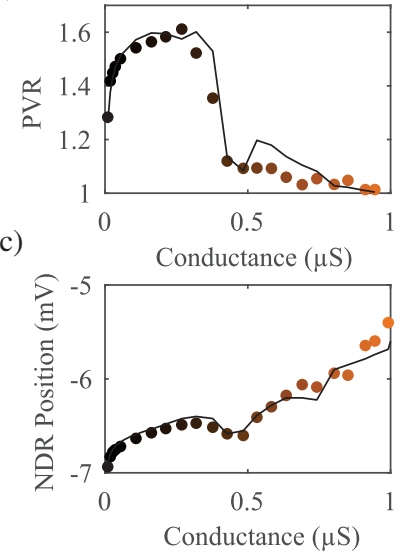

FIG. 2. (a) Conductance-dependent $I(V)$ curves recorded with a spin-polarized tip on the side atom of the Fe trimer (tunnel junction conductance changes from 0.01 to $1.06 \mu \mathrm{S}$ with about $0.105 \mu \mathrm{S}$ per interval and a $1 \mathrm{~T}$ magnetic field). The inset shows the location of the valley and the peak current $\left(I_{v}, I_{p}\right)$. The position of the NDR is defined as the voltage of highest negative slope of the $I(V)$. (b) Peak-to-valley ratio $\left(\mathrm{PVR}=I_{p} / I_{v}\right)$ as a function of set point conductance (colored points, experimental data; solid line, calculation). (c) Position of the NDR as a function of the set point conductance (colored points, experimental data; solid line, calculation). 
the Fe trimer and the rate at which spin-polarized electrons tunnel through the $\mathrm{Fe}$ trimer.

To develop a deeper insight, we model the experiment using a Pauli master equation approach [18,30-40], accounting for the average spin-state occupation of the Fe trimer and the rate of tunneling electrons between the electrodes (STM tip and $\mathrm{Cu}$ substrate) and the Fe trimer. The time evolution of the spin-state population is given as

$$
\frac{d n_{i}(t)}{d t}=\sum_{j}\left[r_{i j} n_{j}(t)-r_{j i} n_{i}(t)\right]
$$

where $n_{i}(t)$ is the average occupation of each spin state and $r_{i j}$ is the transition rate from state $j$ to $i$. The transition rate $r_{i j}=r_{i j}^{s \leftarrow 1 \leftarrow t}+r_{i j}^{t \leftarrow 1 \leftarrow s}+r_{i j}^{t \leftarrow 1 \leftarrow t}+\sum_{a=1}^{3} r_{i j}^{s \leftarrow a \leftarrow s}$ is the sum of all possible transition rates causing the transition from $j$ to $i$. The rates originate from tunneling events through the side atom "1" from the STM tip $(t)$ to the Cu substrate $(s)$ $(s \leftarrow 1 \leftarrow t)$ and vice versa $(t \leftarrow 1 \leftarrow s)$, as well as backscattering into the tip $(t \leftarrow 1 \leftarrow t)$, and from the substrate back into the substrate $(s \leftarrow a \leftarrow s)$ through each of the three $\mathrm{Fe}$ atoms $(a=1,2,3)$ of the $\mathrm{Fe}$ trimer.

The model links the transition rates of tunneling electrons between the STM tip and substrate directly to the rate of transitions between spin states of the Fe trimer. This link originates from the nature of the electron-spin scattering that is well described as Kondo-type electron spin scattering $[32,41,42]$. A scattering Hamiltonian of the form $\hat{H}_{s}=\hat{\vec{S}} \hat{\vec{\sigma}}+u \hat{I}$ is used, where $\hat{\vec{S}}$ is the vector spin operator of the magnetic atom with which the electron interacts, $\hat{\vec{\sigma}}$ is the vector spin operator of the tunneling electron, and $\hat{I}$ is the identity operator accounting for spin-independent scattering with strength $u$. This scattering Hamiltonian was previously found to quantitatively account for elastic and inelastic electron tunneling at magnetic atoms and molecules [18,43-51]. To first order, the transition rates are given by the scattering Hamiltonian's transition matrix elements between the spin states of Fe trimer $|i\rangle$ and $|j\rangle$ [24].

Then, the $I(V)$ curve can be expressed as

$$
I(V)=e \sum_{j, i}\left[r_{i j}^{s \leftarrow 1 \leftarrow t}(V)-r_{i j}^{t \leftarrow 1 \leftarrow s}(V)\right] \tilde{n}_{j}(V),
$$

where $\tilde{n}_{j}(V)$ are the steady-state solutions of Eq. (1). Taking the numerical derivative of the $I(V)$ curves yields the $d I / d V$ spectra, which are fit to the experimental data using least squares optimization [24]. Figures 3(a), 3(c), and 3(d) show that the calculated $I(V)$ curves and $d I / d V$ spectra reproduce the experimental data quantitatively. The calculated tunneling current is the sum of inelastic transitions, $i \neq j$, and elastic transitions, $i=j$. We find that the inelastic tunnel current has a fast onset at bias $|V|>6.4 \mathrm{mV}$ and rises monotonically with an increasing bias magnitude [Fig. 3(a)]. The NDR stems from the elastic current that drops sharply with the onset of inelastic tunneling [Fig. 3(a)].
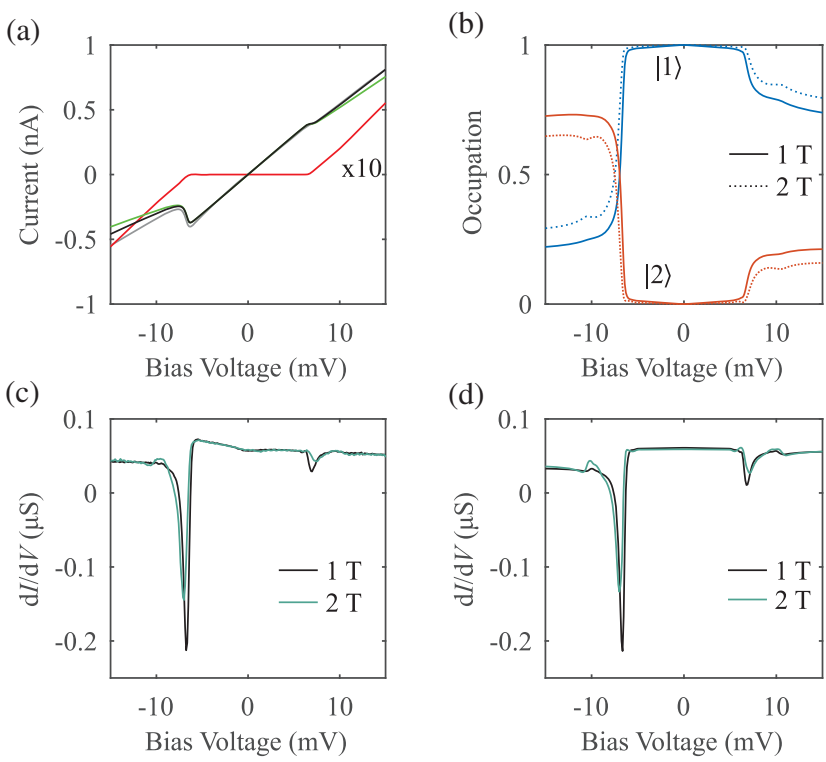

FIG. 3. (a) Calculated (black line) and measured (gray line) spin-polarized $I(V)$ curves on the side atom (set point of $0.054 \mu \mathrm{S}$ at $1 \mathrm{~T}$ ). The elastic (green line) and the inelastic (red line, 10× magnified) current contributions are shown separately. (b) Calculated voltage-dependent occupation $n_{i}(V)$ of the two low-energy states $(|1\rangle$ and $|2\rangle$ ) for a $1 \mathrm{~T}$ (solid lines) and $2 \mathrm{~T}$ (dashed lines) magnetic field. (c) The $d I / d V$ spectra measured at $1 \mathrm{~T}$ (black line) and $2 \mathrm{~T}$ (green line) with a set point of $0.054 \mu \mathrm{S}$. These two spectra are measured with the same spinpolarized tip on the same side atom of the Fe trimer. (d) The correspondingly calculated $d I / d V$ spectra at $1 \mathrm{~T}$ (black line) and $2 \mathrm{~T}$ (green line) with a set point of $0.054 \mu \mathrm{S}$.

This behavior can be understood by the dynamic reconfiguration of the $\mathrm{Fe}$ trimer's spin-state occupation when the tunnel current passes through it. The Fe trimer features two low-energy spin states. They are the Néel-like antiferromagnetic configurations of the $\mathrm{Fe}$ atom magnetic moments and denoted as $|1\rangle$ and $|2\rangle$ in the following. Because of the odd number of magnetic atoms, these states split in magnetic fields by $0.46 \mathrm{meV} / \mathrm{T} \mathrm{[20]}$.

At our measurement conditions ( $0.5 \mathrm{~K}$ temperature, $1 \mathrm{~T}$ or $2 \mathrm{~T}$ magnetic field), the thermal occupation of state $|2\rangle$ is negligible. As the sample bias decreases from zero, the occupation of state $|2\rangle$ increases only slowly, since the probability for a direct transition between the two states is small. All electric current is carried by elastic spindependent tunneling. When the bias drops below $-6.8 \mathrm{mV}$, state $|2\rangle$ becomes populated rapidly and dynamically locked in this state [Fig. 3(b)]. State $|2\rangle$ has a markedly smaller transition matrix element for elastic tunneling than state $|1\rangle$ because of the spin-polarized STM tip. As such, the rapid occupation change quickly decreases the conductance, and the net current through the Fe trimer drops. The effective magnetoresistance between the spin-polarized STM tip and Fe trimer is therefore bias dependent and causes the NDR. 
Our calculations show that the occupation change at $-6.8 \mathrm{mV}$ is driven by an efficient two-step transition through intermediate states where the spin momentum of the Fe trimer is decreased by $1 \hbar$. The intermediate states, located $6.8 \mathrm{meV}$ above the ground state, are energetically unfavorable, and they do not become significantly populated because of magnetic anisotropy and the interatomic exchange energy of the Fe trimer [24].

The presence of this process is corroborated by the asymmetry of the $I(V)$ curves with respect to bias reversal and their variation with the magnetic field. Because of spinmomentum conservation, tunneling electrons exciting the two-step transition must change their spin by $+1 \hbar$, i.e., from $\left\langle\sigma_{z}\right\rangle=-1 / 2$ to $\left\langle\sigma_{z}\right\rangle=+1 / 2$. With a negative bias, electrons tunnel into $\sigma_{z}=+1 / 2$ states of the tip, whereas they tunnel out of $\sigma_{z}=-1 / 2$ states for a positive bias, since the tip is strongly spin polarized. Hence, inelastic transitions are more frequent at a negative bias and generate a more pronounced dip in $d I / d V$. In addition, the transition energy between the intermediate states and states $|1\rangle$ and $|2\rangle$ depends on the magnetic field because of the difference in their net magnetic moment. Upon increasing the magnetic field from $1 \mathrm{~T}$ to $2 \mathrm{~T}$, the NDR feature shifts to a higher energy, consistent with the difference in Zeeman energy between the states [Figs. 3(c) and 3(d)]. Surprisingly, the PVR, i.e., the NDR amplitude, also reduces. This is caused by an increase of the transition rate from state $|2\rangle$ into the intermediate states which reduces the occupation of $|2\rangle$ compared to $1 \mathrm{~T}$ [Fig. 3(b)].

This sensitivity of the NDR to magnetic fields points to the importance of considering the coupling between the spin-polarized tip and the Fe trimer in more detail. For the tunnel current to flow, wave functions of the Fe trimer and the tip must overlap. Since the tip apex is spin polarized and magnetic, the same overlap may induce a magnetic exchange interaction [52]. Indeed, the reduction of the PVR for junction conductances in excess of $0.27 \mu \mathrm{S}$ [Fig. 2(b)] cannot be explained by the increasing tunneling rates alone. Antiferromagnetic interaction between the spin-polarized STM tip and Fe trimer must be included in order achieve quantitative fits to the $I(V)$ curves [24].

The interaction reduces the energy separation between states $|1\rangle$ and $|2\rangle$ and increases the transition probability. For an applied magnetic field of $1 \mathrm{~T}$ and junction conductances larger than $0.27 \mu \mathrm{S}$, this leads to a significant population of state $|2\rangle$ even at $|V|<6.8 \mathrm{mV}(0.5 \mathrm{~K})$. Therefore, upon reaching the excitation threshold, the change in the state population is less pronounced compared to a smaller junction conductance and the NDR is reduced [reduced PVR in Fig. 2(b)]. In addition, the energy separation to the intermediate spin state of the two-step excitation reduces, which is observed as a position shift of the NDR to a lower bias [Fig. 2(c)]. The center of the NDR shifts from $-6.8 \mathrm{mV}$ for a junction conductance of $0.01 \mu \mathrm{S}$ to $-5.6 \mathrm{mV}$ at a conductance of $0.95 \mu \mathrm{S}$. Notably, at a junction conductance of $0.43 \mu \mathrm{S}$ states $|1\rangle$ and $|2\rangle$ reverse order, because the coupling with the spin-polarized tip becomes stronger than the external magnetic field and all dynamic processes reverse: The magnetoresistance for elastic tunneling is now large at a small bias and does not increase further when the bias magnitude exceeds $5.4 \mathrm{mV}$. Consequently, the NDR at a negative bias disappears, and a pronounced conductance peak appears at a positive bias [Fig. 4(a)]. Upon increasing the magnetic field to $2 \mathrm{~T}$, the conductance-dependent $d I / d V$ spectra change slightly [Figs. 4(a) and 4(b)]. For a $2 \mathrm{~T}$ magnetic field, a stronger coupling strength with the spin-polarized tip is needed to reverse the dynamic processes. For that reason, the conductance dip on the negative bias side disappears at a larger junction conductance in comparison to the $1 \mathrm{~T}$ external magnetic field case [Figs. 4(a) and 4(b)].

Since the tunnel current and exchange interaction typically decay exponentially with the tunneling gap size $[20,53]$, we consider a conductance-dependent Ising interaction of the form $J_{t}(\sigma) \sim \sigma^{\kappa}$, where $\kappa$ is the ratio of decay constants for tunneling and magnetic interaction. By fitting the conductance-dependent $d I / d V$ spectra with 1 and $2 \mathrm{~T}$
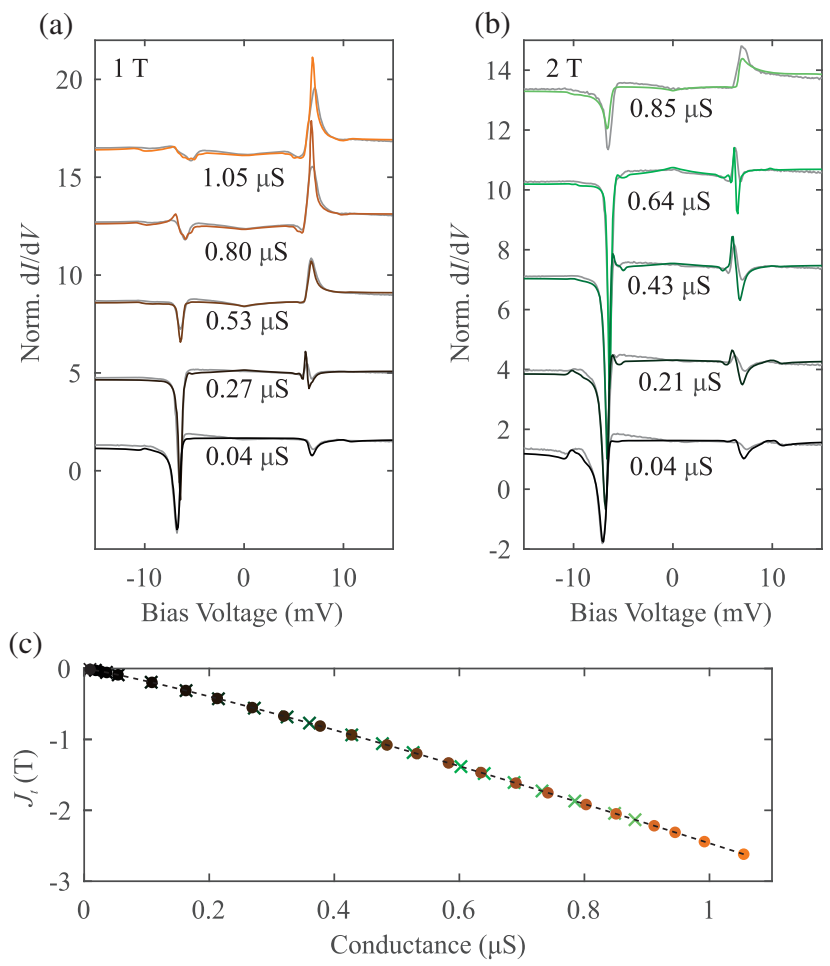

FIG. 4. (a), (b) The measured conductance-dependent $d I / d V$ spectra (gray lines) with a 1 and $2 \mathrm{~T}$ magnetic field applied, respectively. The calculated $d I / d V$ spectra are shown as colored lines. For visibility, the spectra are normalized to the set point conductance and are offset. (b) The strength of the tip interaction as a function of the conductance set point as extracted by fitting the measured conductance-dependent $d I / d V$ spectra. Colored markers indicate the junction conductances used for the fit (dots, $1 \mathrm{~T}$; crosses, $2 \mathrm{~T}$ ). 
magnetic fields simultaneously, we find $\kappa=(1.1 \pm 0.1)$ reproduces both measurements with a high accuracy [Fig. 4(c)]. Hence, the magnetic interaction and tunnel current are carried by wave functions with a similar decay into a vacuum, emphasizing that both effects are of equal importance for the spin-based NDR.

In conclusion, we report spin-based NDR from an antiferromagnetically coupled few-atom spin chain in a spin-polarized tunneling junction. The NDR occurs at a low bias magnitude of $7 \mathrm{mV}$ and is caused by a two-step excitation that drives fast transitions between the two Néel-like spin states of the spin chain and dynamically locks it in a configuration with a large magnetoresistance. The NDR varies nonmonotonically with the junction conductance between the spin-polarized tip and spin chain. We attribute this behavior to a competition between the dynamic locking by inelastic electron tunneling that produces NDR and the static magnetic exchange interaction that prevents it. Our measurements suggest that both effects are mediated by similar wave functions and likely linked inseparably. This spin-based NDR will be observable for other magnetic nanoobjects with discrete spin states, such as singlemolecule magnets [17] and nanoferromagnets [47]. As it occurs at very low voltages, on the order of the magnetic anisotropy, and at low currents, it may prove useful for applications in low-current spintronic devices. Because of the sensitivity of this NDR to magnetic interaction, the effect has the potential as the basis for an atomic-scale magnetic sensor.

The authors thank E. Weckert and H. Dosch (Deutsches Elektronen-Synchrotron, Germany) for providing lab space. This project has received funding from the European Research Council (ERC) under the European Union's Horizon 2020 research and innovation program (ERC-2014-StG-633818-dasQ). S. R.-P. acknowledges a fellowship from the Studienstiftung des deutschen Volkes (German Academic Scholarship Foundation) and J. A. J. B. postdoctoral fellowships from the Alexander von Humboldt foundation and the Natural Sciences and Engineering Research Council of Canada.

S. R.-P. and S. Y. contributed equally to this work.

*steffen.rolf-pissarczyk@mpsd.mpg.de

†yanshch@shanghaitech.edu.cn

*sebastian.loth@fmq.uni-stuttgart.de

[1] S. A. Wolf, D. D. Awschalom, R. A. Buhrman, J. M. Daughton, S. von Molnár, M. L. Roukes, A. Y. Chtchelkanova, and D. M. Treger, Science 294, 1488 (2001).

[2] I. Zutic, J. Fabian, and S. Das Sarma, Rev. Mod. Phys. 76, 323 (2004).

[3] L. Bogani and W. Wernsdorfer, Nat. Mater. 7, 179 (2008).

[4] L. Esaki, Rev. Mod. Phys. 46, 237 (1974).

[5] M. Grundmann, The Physics of Semiconductors (SpringerVerlag, Berlin, 2006).
[6] J. Chen, M. A. Reed, A. M. Rawlett, and J. M. Tour, Science 286, 1550 (1999).

[7] M. L. Perrin, R. Frisenda, M. Koole, J. S. Seldenthuis, G. A. Celis, H. Valkenier, J. C. Hummelen, N. Renaud, F. C. Grozema, J. M. Thijssen, D. Dulié, and S. J. van der ZantHerre, Nat. Nanotechnol. 9, 830 (2014).

[8] B. Xu and Y. Dubi, J. Phys. Condens. Matter 27, 263202 (2015).

[9] J. Gaudioso, L. J. Lauhon, and W. Ho, Phys. Rev. Lett. 85, 1918 (2000).

[10] N. J. Tao, Nat. Nanotechnol. 1, 173 (2006).

[11] B. Warner, F. El Hallak, H. Prüser, J. Sharp, M. Persson, A. J. Fisher, and C. F. Hirjibehedin, Nat. Nanotechnol. 10, 259 (2015).

[12] M. Rashidi, M. Taucer, I. Ozfidan, E. Lloyd, M. Koleini, H. Labidi, J. L. Pitters, J. Maciejko, and R. A. Wolkow, Phys. Rev. Lett. 117, 276805 (2016).

[13] D. Weinmann, W. Häusler, and B. Kramer, Phys. Rev. Lett. 74, 984 (1995).

[14] K. Ono, D. G. Austing, Y. Tokura, and S. Tarucha, Science 297, 1313 (2002).

[15] M. Pioro-Ladrière, M. Ciorga, J. Lapointe, P. Zawadzki, M. Korkusiński, P. Hawrylak, and A. S. Sachrajda, Phys. Rev. Lett. 91, 026803 (2003).

[16] H. B. Heersche, Z. de Groot, J. A. Folk, H. S. J. van der Zant, C. Romeike, M. R. Wegewijs, L. Zobbi, D. Barreca, E. Tondello, and A. Cornia, Phys. Rev. Lett. 96, 206801 (2006).

[17] F. Elste and C. Timm, Phys. Rev. B 73, 235305 (2006).

[18] S. Loth, K. von Bergmann, M. Ternes, A. F. Otte, C. P. Lutz, and A. J. Heinrich, Nat. Phys. 6, 340 (2010).

[19] S. Loth, S. Baumann, C. P. Lutz, D. M. Eigler, and A. J. Heinrich, Science 335, 196 (2012).

[20] S. Yan, D.-J. Choi, J. A. J. Burgess, S. Rolf-Pissarczyk, and S. Loth, Nat. Nanotechnol. 10, 40 (2015).

[21] S. Yan, D.-J. Choi, J. A. J. Burgess, S. Rolf-Pissarczyk, and S. Loth, Nano Lett. 15, 1938 (2015).

[22] M. Bode, Rep. Prog. Phys. 66, 523 (2003).

[23] R. Wiesendanger, Rev. Mod. Phys. 81, 1495 (2009).

[24] See Supplemental Material at http://link.aps.org/ supplemental/10.1103/PhysRevLett.119.217201 for details on the experimental setup, the rates in the Pauli master equation and spin Hamiltonian, the fit routine, the currentdependent $I(V)$ curves at a 1 and 2 Texternal magnetic field, the time evolution of the state occupation, the resistance as a function of the voltage, and the spin-polarized $I(V)$ curve and $d I / d V$ spectrum recorded on the center atom of the $\mathrm{Fe}$ trimer, which includes Refs. [20,21,25].

[25] W. Nolting and A. Ramakanth, Quantum Theory of Magnetism (Springer, New York, 2009).

[26] J. Repp, G. Meyer, S. M. Stojković, A. Gourdon, and C. Joachim, Phys. Rev. Lett. 94, 026803 (2005).

[27] A. J. Heinrich, J. A. Gupta, C. P. Lutz, and D. M. Eigler, Science 306, 466 (2004).

[28] C. F. Hirjibehedin, C. P. Lutz, and A. J. Heinrich, Science 312, 1021 (2006).

[29] S. Baumann, F. Donati, S. Stepanow, S. Rusponi, W. Paul, S. Gangopadhyay, I. G. Rau, G. E. Pacchioni, L. Gragnaniello, M. Pivetta, J. Dreiser, C. Piamonteze, C. P. Lutz, R. M. Macfarlane, B. A. Jones, P. Gambardella, A. J. Heinrich, and H. Brune, Phys. Rev. Lett. 115, 237202 (2015). 
[30] S. Loth, C. P. Lutz, and A. J. Heinrich, New J. Phys. 12, 125021 (2010).

[31] F. D. Novaes, N. Lorente, and J.-P. Gauyacq, Phys. Rev. B 82, 155401 (2010).

[32] M. Ternes, New J. Phys. 17, 063016 (2015).

[33] A. A. Khajetoorians, B. Baxevanis, C. Hübner, T. Schlenk, S. Krause, T. O. Wehling, S. Lounis, A. Lichtenstein, D. Pfannkuche, J. Wiebe, and R. Wiesendanger, Science 339, 55 (2013).

[34] B. W. Heinrich, L. Braun, J. I. Pascual, and K. J. Franke, Nat. Phys. 9, 765 (2013).

[35] B. W. Heinrich, L. Braun, J. I. Pascual, and K. J. Franke, Nano Lett. 15, 4024 (2015).

[36] F. Delgado and J. Fernández-Rossier, Phys. Rev. B 82, 134414 (2010).

[37] M. Muenks, P. Jacobson, M. Ternes, and K. Kern, Nat. Commun. 8, 14119 (2016).

[38] L. Wei, W. Rui-Qiang, H. Liang-Bin, and Y. Mou, Chin. Phys. B 22, 047201 (2013).

[39] H. Xie, Q. Wang, H.-B. Xue, H. Jiao, and J.-Q. Liang, J. Appl. Phys. 113, 213708 (2013).

[40] C. Zhang, H. Yao, Y.-H. Nie, and J.-Q. Liang, AIP Adv. 6, 115202 (2016).

[41] J. A. Appelbaum, Phys. Rev. 154, 633 (1967).
[42] F. Delgado, J. J. Palacios, and J. Fernández-Rossier, Phys. Rev. Lett. 104, 026601 (2010).

[43] G.-H. Kim and T.-S. Kim, Phys. Rev. Lett. 92, 137203 (2004).

[44] J. Fernández-Rossier, Phys. Rev. Lett. 102, 256802 (2009).

[45] N. Lorente and J.-P. Gauyacq, Phys. Rev. Lett. 103, 176601 (2009).

[46] M. Ternes, Prog. Surf. Sci. 92, 83 (2017).

[47] A. Spinelli, B. Bryant, F. Delgado, J. Fernández-Rossier, and A. F. Otte, Nat. Mater. 13, 782 (2014).

[48] B. Bryant, A. Spinelli, J. J. T. Wagenaar, M. Gerrits, and A. F. Otte, Phys. Rev. Lett. 111, 127203 (2013).

[49] S. Kahle, Z. Deng, N. Malinowski, C. Tonnoir, A. FormentAliaga, N. Thontasen, G. Rinke, D. Le, V. Turkowski, T. S. Rahman, S. Rauschenbach, M. Ternes, and K. Kern, Nano Lett. 12, 518 (2012).

[50] A. Hurley, N. Baadji, and S. Sanvito, Phys. Rev. B 84, 035427 (2011).

[51] J.-P. Gauyacq, S. M. Yaro, X. Cartoixà, and N. Lorente, Phys. Rev. Lett. 110, 087201 (2013).

[52] P. Ferriani, C. Lazo, and S. Heinze, Phys. Rev. B 82, 054411 (2010).

[53] R. Schmidt, C. Lazo, U. Kaiser, A. Schwarz, S. Heinze, and R. Wiesendanger, Phys. Rev. Lett. 106, 257202 (2011). 